\title{
Effect of the feed substrate concentration on the dynamic performance of the bioethanol fermentation process using Zymomonas mobiliz
}

\begin{abstract}
In this work, a structured verified nonlinear mathematical model for a single continuous fermenter of bioethanol production using Zymomonas mobiliz is developed to investigate the effect of the feed substrate concentration, which is taken as the main bifurcation parameter for performing a bifurcation analysis. The chaotic, oscillatory, and steady-state behaviors of the fermentation system are investigated. It is found that the system is dominated by oscillatory behavior in the medium gravity (MG) region $(80.76<$ Cso < 213.6), while it is dominated by stable steady state outside this range; however, at high Cso and low dilution rate, the chaotic behavior dominates the system. It is found that the fermentation system accomplishes the maximum averages of substrate conversion and bioethanol yield in the range of $80.76<$ Cso $<97 \mathrm{~g} / \mathrm{L}$. These results are in agreement with the experimental results in the literature.
\end{abstract}

Keyword: Zymomonas mobiliz; Feed substrate concentration; Fermentation; Bioethanol 